\title{
Iterative approximations of common fixed points with simulation results in Banach spaces
}

\author{
Ashis Bera ${ }^{1}$, Ankush Chanda ${ }^{2}$, and Lakshmi Kanta Dey ${ }^{1}$ \\ ${ }^{1}$ National Institute of Technology Durgapur \\ ${ }^{2}$ VIT University
}

December 26, 2020

\begin{abstract}
In this article, we propose the Abbas-Nazir three step iteration scheme and employ the algorithm to study the common fixed points of a pair of generalized $\$ \backslash$ alpha-Reich-Suzuki non-expansive mappings defined on a Banach space. Moreover, we explore a few weak and strong convergence results concerning such mappings. Our findings are aptly validated by non-trivial and constructive numerical examples and finally, we compare our results with that of the other noteworthy iterative schemes utilizing MATLAB $\$ 2017 \$$ a software. However, we perceive that for a different set of parameters and initial points, the newly proposed iterative scheme converges faster than the other well-known algorithms. To be specific, we give an analytic proof of the claim that the novel iteration scheme is also faster than that of Liu et al.
\end{abstract}

\section{Hosted file}

Paper.pdf available at https://authorea.com/users/385240/articles/500730-iterativeapproximations-of-common-fixed-points-with-simulation-results-in-banach-spaces 